\title{
CFD and Volume Fraction Analysis of Closed Loop Pulsating Heat Pipe(CLPHP)
}

\author{
Mr. Mohd Aijaz ul Haque ${ }^{1}$, Dr. Md Azizuddin ${ }^{2}$,Mr.Md Khalid Rehman ${ }^{3}$ \\ ${ }^{1,3}$ (Assistant Professor, Mechanical Engineering Department, Deccan College of Engineering and \\ Technology,India \\ ${ }_{2}^{2}$ (Professor, Mechanical Engineering Department, Deccan College of Engineering and Technology, India
}

\begin{abstract}
The Closed loop pulsating heat pipes (CLPHPS) are complex heat transfer devices having a strong thermo hydrodynamic coupling governing the thermal performance. Attempts are made to design, fabricate and test a closed loop pulsating heat pipe. In this experiment three different configurations of copper capillary tubes having internal diameters the total length and diameter of the channel is $540 \mathrm{~mm}$ and $1.95 \mathrm{~mm}$ respectively; and the pipe is made of copper. In this work, acetone-acetone vapor is taken as the working fluid for analysis. The experiments are conducted on vertical orientations for different heat loads as working fluids for fill ratio viz. $60 \%$, the temperature distribution across the heat pipe was measured and recorded using thermocouples. The performance parameters such as temperature difference between evaporator and condenser, thermal resistance and the overall heat transfer coefficient are evaluated. The experimental results demonstrate the heat transfer characteristics, lower thermal resistance and higher heat transfer coefficient of PHP are found to be better at a fill ratio of 60\% for various heat input and also the thermal performance of set up of pulsating heat pipe gives better result over the uniform diameter CLPHP the CFD analysis is done with the help of Ansys 15.0 software.
\end{abstract}

Keywords: Closed Loop Systems,CLPHP, CFD, Pulsating Heat Pipes, Volume Fraction Analysis

\section{Introduction}

Close Loop Pulsating heat pipes (CLPHPs) typically suited for microelectronics cooling consists of a plain meandering tube of capillary dimensions with many U-turns and joined end to end. The pipe is first evacuated and then filled partially with a working fluid. If the diameters of Close Loop Pulsating heat pipe is not too large, the fluid distributes itself into an arrangement of liquid slugs separated by vapor bubbles. One end of this tube bundle receives heat transferring it to the other end by a pulsating action of the liquid-vapor/slugbubble system. The liquid and vapor slug/bubble transport is caused by the thermally induced pressure pulsations inside the device and no external mechanical power is required. The type of fluid and the operating pressure inside the pulsating heat pipe depend on the operating temperature of the heat pipe.

The region between evaporator and condenser is adiabatic. The heat is transfer from evaporator to condenser by the means of pulsating action of vapor slug and liquid slug. This pulsation appears as a nonequilibrium chaotic process, whose continuous operation requires non-equilibrium conditions inside the tube in some of the parallel channels. For Close Loop Pulsating heat pipes (CLPHPs), no external power source is needed to either initiate or sustain the fluid motion or the transfer of heat. The purpose of this project is to understand how CLPHPs operate and to be able to understand how various parameters (geometry, fill ratio, materials, working fluid, etc.) affect its performance. Understanding its operation is further complicated by the non-equilibrium nature of the evaporation and condensation process, bubble growth and collapse and the coupled response of the multiphase fluid dynamics among the different channels.

\subsection{Heat Pipe}

The heat pipe has two region i.e. evaporator and condenser. There is adiabatic region which separates condenser and evaporator. The heat pipe has wall, the wick structure and the space for the working fluid.



Fig. 1.1 Schematic diagram of Heat pipe 


\subsection{Working principle of Heat Pipe}

The heat is absorbed in the evaporator region and is carried out through the pipe by the evaporation of the fluid by absorbing the heat. The high temperature vapor movies toward the condenser by the action of buoyancy force. At the condenser it rejects the heat by convection and coverts into liquid droplets. These droplets move to the evaporator due to gravity though the wick material.

\subsection{Objective of the present work}

\section{Objective And Methodology}

The objective of the present work is to study the performance of closed loop pulsating heat pipe with a single turn using ANSYS FLUENT 15.0. This work includes enhancement of the heat transfer rate of a PHP by varying load conditions in evaporator with different filling ratios of working fluid. Also to find the filling ratio that gives minimum thermal resistance and maximum heat transfer rate and the results obtained by CFD analysis are to be compared with experimental paper results of R. Naik, V. Varadarajan, G. Pundarika and K. R. Narasimha et al [8]. Acetone is used as working fluid.

\subsection{Methodology}

First of all the literature survey is done on the operation and performance of pulsating heat pipe. Relevant recommendations made on the papers and journals are considered to come up with this present work. In this regard, the paper by R. Naik, V. Varadarajan , G. Pundarika and K. R. Narasimha on " Experimental Investigation and Performance Evaluation of a Closed Loop Pulsating Heat Pipe " et al [8] is taken as the reference paper for the basic geometry, operating conditions and performance parameter. This paper has presented on the experimental analysis.

Later the tools for solving the model are decided and worked on. After setting suitable schemes, simulation is run till the desired convergence criteria is achieved. The data from the result is categorized and then compared with the experimental work of R. Naik, V. Varadarajan, G. Pundarika and K. R. Narasimha et al [8]. The overall steps used in this research are summarized in a flow chart diagram as shown in figure 2.1.

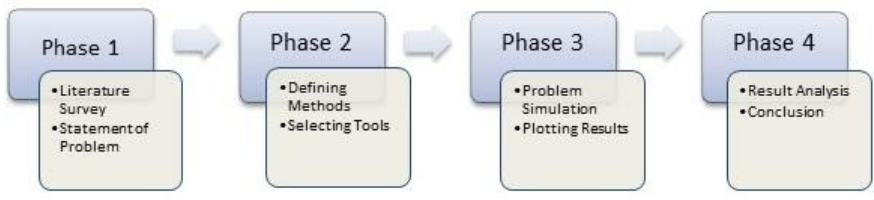

Fig. 2.1 Methodology chart for the study The overall steps used in this research are

First phase: First the literature survey is done on the operation and performance of pulsating heat pipe. These literatures are considered to come up with this present work and the experimental paper by R. Naik, V. Varadarajan, G. Pundarika and K. R. Narasimha et al [8] is taken as the reference for the basic geometry of PHP. Geometry is created in ANSYS FLUENT 15.0. Meshing is done for geometry and names are given to meshing sections which makes it easy while defining domain and giving boundary conditions in the FLUENT.

Second phase: In this phase various methods and tools are selected in ANSYS FLUENT setup and FLUENT solution for the present analysis.

ANSYS FLUENT setup: The methods and tools selected in ANSYS FLUENT setup are transient analysis, multiphase models, volume fraction model, effect of courant number on analysis, turbulence model, material and phase selection, boundary conditions like momentum and thermal properties and in cell conditions operating pressure, temperature and fluid density are selected. And this is an important phase in this CFD analysis.In FLUENT solution required solution methods, solution controls are selected and in solution initialization patch is done. In run calculation timestep size and number of timesteps are given to obtain required convergence criteria.

Third phase: In Run calculation timestep size and number of timesteps are given, so the simulation is done till the convergence criteria is obtained. At this stage results are taken like temperatures at evaporator section and condenser section, from these temperatures thermal resistance and heat transfer coefficients are calculated for varying heat loads. And the volume fraction variations in PHP at different timesteps are observed.

Fourth phase: The results which are obtained by CFD analysis are compared with the experimental by R. Naik, V. Varadarajan , G. Pundarika and K. R. Narasimha et al [10], and the thermal resistance and heat transfer coefficients are compared at different fill ratios. 


\subsection{CFD Procedure}

\section{Cfd Modelling Of Sinfgle Loop Php}

Based on control volume method, 3-D analysis of fluid flow and heat transfer for the tube is done on ANSYS FLUENT 15.0 software. The PHP has a three sections viz., evaporator, adiabatic section and condenser sections.

Evaporator Region Length $=185 \mathrm{~mm}$

Condenser Region length $=195 \mathrm{~mm}$.

Adiabatic Region Length $=160 \mathrm{~mm}$.

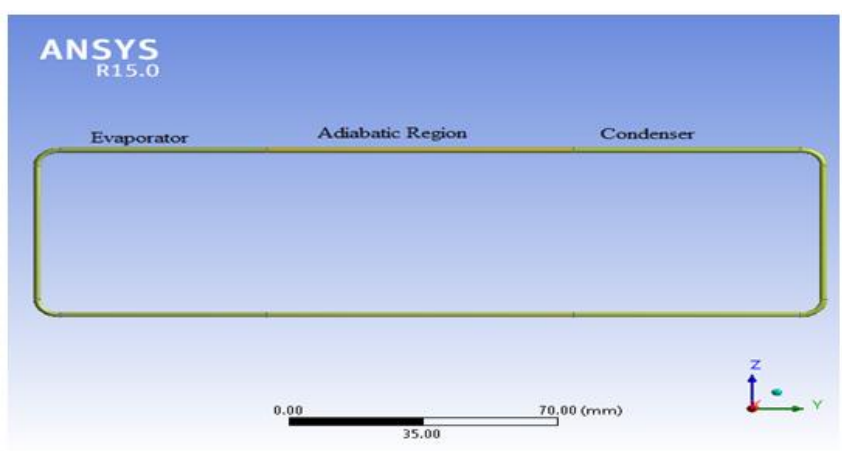

Fig3.1 Geometry created in ANSYS 15.0

\subsubsection{Geometry Creation}

In this present work for creating a geometry the reference of geometry design of closed loop pulsating heat pipe (CLPHP) is taken into consideration as suggested by R. Naik, V. Varadarajan, G. Pundarika and K. R. Narasimhain et al [10]. The total length and diameter of the channel is $540 \mathrm{~mm}$ and $1.95 \mathrm{~mm}$ respectively and the pipe is made of copper.

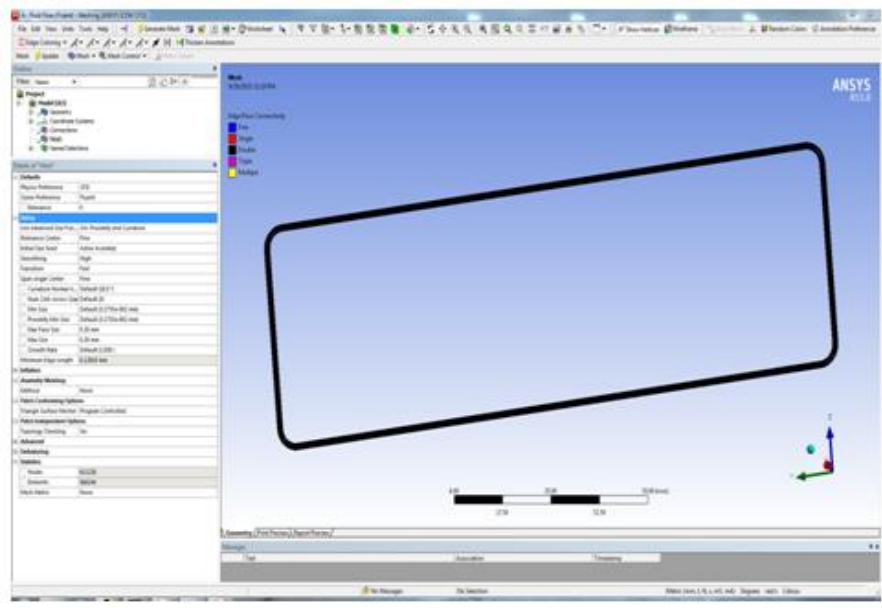

Fig 3.2 Geometry of PHP meshed in ANSYS15.0

\begin{tabular}{|l|l|l|l|l|}
\hline Description & Symbol & Acetone liquid & Acetone vapor & Units \\
\hline Density & $\rho$ & 791 & 2.37 & $\mathrm{~kg} / \mathrm{m} 3$ \\
\hline Dynamic Viscosity & $\mu$ & 0.000331 & 0.000009 & $\mathrm{~kg} / \mathrm{ms}$ \\
\hline Specific Heat & $\mathrm{Cp}$ & 2160 & 1386 & $\mathrm{~J} / \mathrm{kg} \mathrm{K}$ \\
\hline Thermal Conductivity & $\mathrm{k}$ & 0.18 & 0.0143 & $\mathrm{~W} / \mathrm{m} \mathrm{K}$ \\
\hline
\end{tabular}

Table 3.1 Property Values for working fluid

\subsubsection{Meshing}

In addition to the automated settings, ANSYS Meshing provides additional control with the option to specify combinations of point controls, edge controls, surface controls and/or body controls. Each one of these has its own options and can be used to influence the mesh in different ways. In this case, the automatic method for mesh shape is selected; however the sizing for the mesh is done manually. The minimum and maximum mesh sizes are chosen to be $0.0002 \mathrm{~m}$ as shown in Fig 3.2 above. With this control setting, meshing is generated with 6,11,226 number of nodes and 5,66,244 number of elements. 


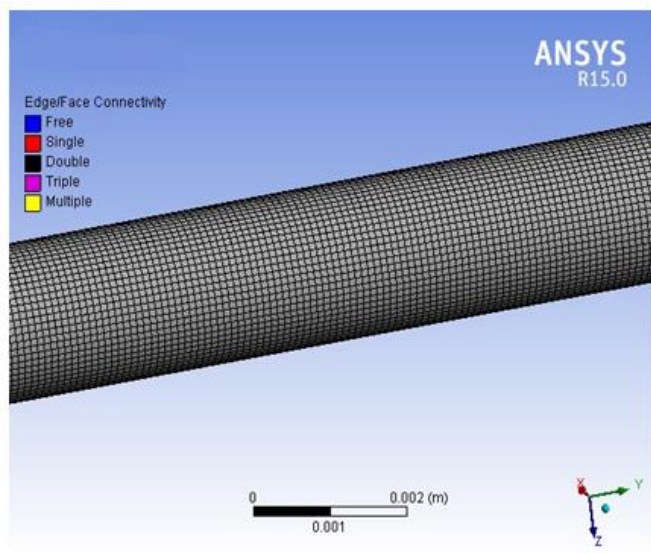

Fig3.3 A Section of Geometry meshed with ANSYS

The Fig.3.3 shows the grid of a section of geometry. It depicts that the domain is meshed with rectangular cells. The quadrilateral grid is generated on all the faces.In meshing the sections of the geometry are given names as evaporator, adiabatic region and condenser section, which makes it easy while defining domain and giving boundary conditions in the set up section of FLUENT

3.2 Turbulence Model: One of the most prominent turbulence models, the $k$-epsilon model, has been implemented in this analysis as is done in most of the general purpose CFD codes and is considered the industry standard model.. For general purpose simulations, the $k$-epsilon offers a good compromise in terms of accuracy and robustness.

3.3Governing Equations: Applying boundary conditions, the governing equations for convective heat transfer are as follows: The critical Bond number criterion gives the tentative design rule for the diameter

$$
\begin{gathered}
(\text { Bo })_{\text {crit }}^{2} \approx \frac{D^{2}\left(\rho_{\text {liq }}-\rho_{\text {vap })}\right)}{\sigma} \approx 4 \\
D_{\text {crit }} \approx 2 . \sqrt{\frac{\sigma}{g \cdot\left(\rho_{\text {liq }}-\rho_{\text {vap }}\right)}}
\end{gathered}
$$

\section{Results And Discussions}

4.1Variation of Thermal Resistance: The Thermal Resistance of PHP is calculated by equation 4.1. $\mathrm{R}=\frac{T_{\varepsilon}-T_{C}}{Q}(\mathrm{k} / \mathrm{w}) \rightarrow 4.1$ Where: $\mathrm{Q}=$ heat flux in $(\mathrm{w}), T_{\varepsilon}=$ Evaporator Temperature in $\mathrm{k}$

$T_{c}=$ Condenser Temperature in $\mathrm{k}$
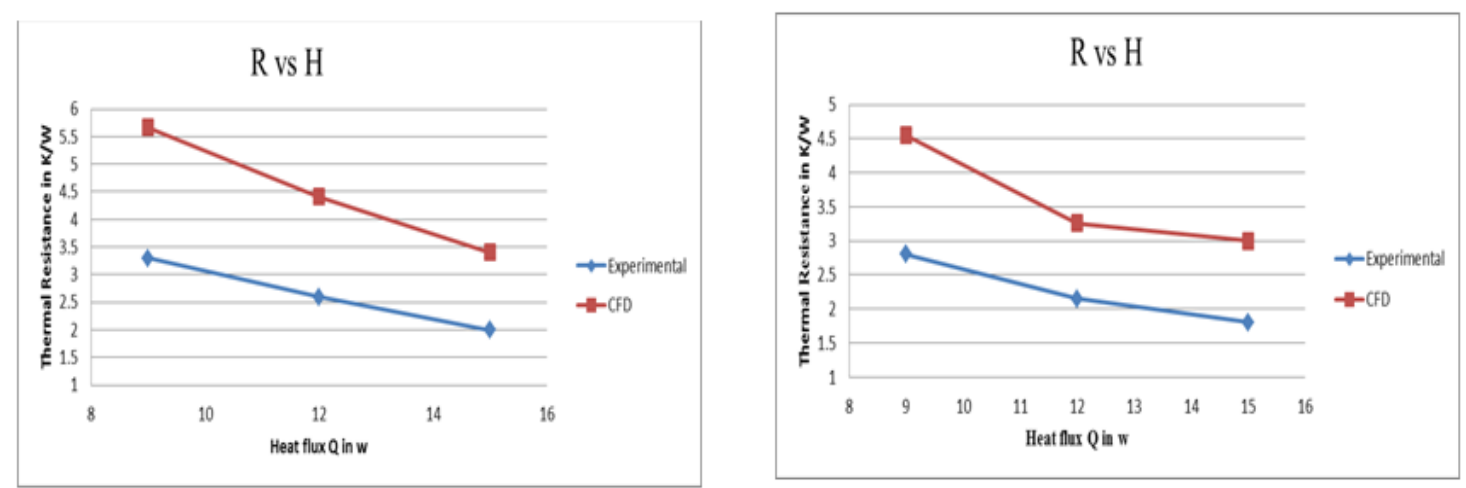

Fig4.1 CFD and Experimental results at 60\% volume fraction Fig4.2 CFD and Experimental results at 80\% volume fraction

Above Figures 4.1 and 4.2 are the comparison graphs between CFD and Experimental results for variation in thermal resistance with heat inputs at $60 \%$ and $80 \%$ fill ratios. It is inferred from the graph is that the Thermal resistance is decreasing with increase in heat flux. The temperature difference between evaporator and condenser decreases with increase in heat input. As the movement of the fluid is very slow at lower heat input which is associated with lot of fluctuations, the temperature difference between evaporator and condenser is higher at lower heat input. So the Thermal resistance is decreasing with increase in heat load. 


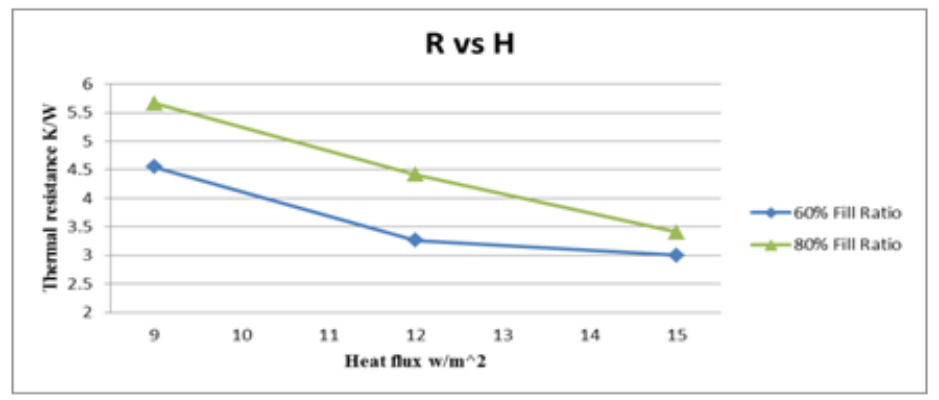

Fig 4.3 Thermal Resistance at different Volume Fractions

Above Fig 4.3 shows the variation of thermal resistance with heat load for Acetone working fluid for different fill ratios. From the figure it is clear that the thermal resistance decreases with increase in heat input. The fill ratio of $60 \%$ exhibits the lower values of thermal resistance compared to higher fill ratio of $80 \%$. As the temperature difference between evaporator and condenser is less at a fill ratio of $60 \%$, the magnitude of thermal resistance is also less. This shows that the heat transfer characteristics in a PHP are better at $60 \%$ fill ratio.

\subsection{Variation of Heat Transfer Coefficient}

The heat transfer coefficient of a PHP is calculated using equation 4.2.

$$
\begin{aligned}
& \mathrm{h}= \frac{Q}{A_{s}\left(T_{e}-T_{c}\right)}\left(\mathrm{w} / m^{2} K\right) \rightarrow 4.2 \text { where: } \mathrm{Q}=\text { heat flux in }(\mathrm{w}), T_{e}=\text { Evaporator Temperature in }(\mathrm{K}) \\
& T_{c}=\text { Condenser Temperature in }(\mathrm{K}) \quad A_{s}=\text { Evaporator surface area in }\left(\mathrm{m}^{2}\right)
\end{aligned}
$$

Figures $4.4 \& 4.5$ are the comparison graphs between CFD and Experimental results for variation in heat transfer coefficient with heat flux at $60 \%$ and $80 \%$ fill ratios. It is inferred from the graphs is that the heat transfer coefficient is increasing with increase in heat flux.

Fig 4.6 is a comparison graph for variation in heat transfer coefficient with heat flux at different volume fractions. It is inferred from the graph is that the heat transfer coefficient is high at $60 \%$ volume fraction as compared with $80 \%$ volume fraction

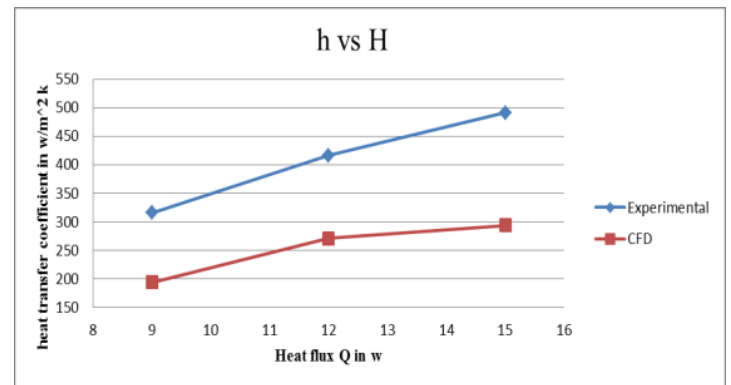

Fig 4.4 CFD and Experimental results at $60 \%$ volume fraction



Fig 4.5 CFD and Experimental results at $80 \%$

4.3Volume fraction contours of PHP:The contours of volume fraction of acetone-liquid, acetonevapour and air in single loop pulsating heat pipe for different time steps are plotted.. Here the first three contours ie., Fig 4.7 to 4.9 are the volume fraction contours of acetone-liquid, acetone-vapour and air in PHPat the initial condition for $60 \%$ filling ratio.

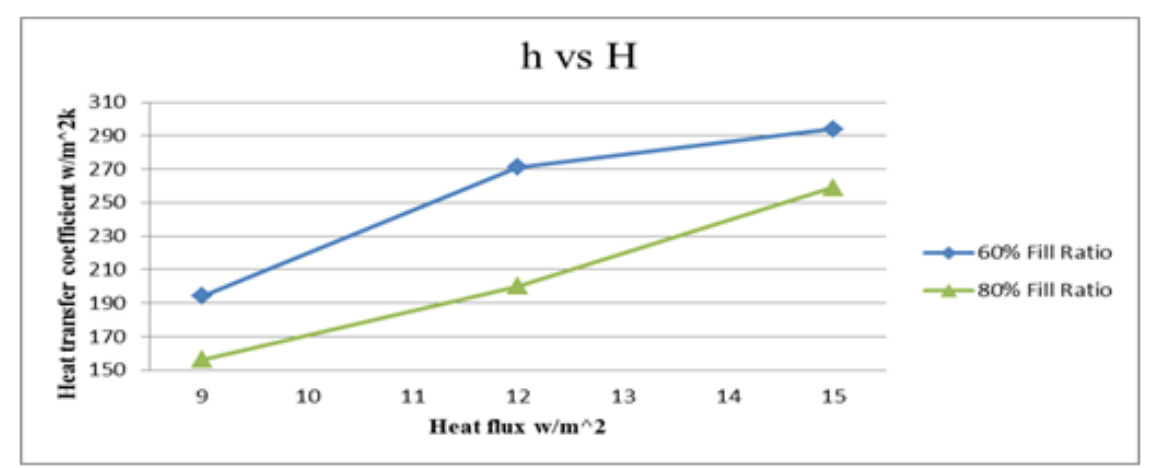

Fig 4.6 Heat transfer coefficient's at different volume fractions 
This clearly shows that the PHP is filled with $60 \%$ of acetone fluid before heat load is given to the evaporator and remaining $40 \%$ of PHP has air in it, this contour of air is shown in Fig 4.8. And at initial condition there is no acetone- vapour in the PHP, this acetone-vapour contours are shown in fig 4.9. These volume fraction contours at initial condition are same for different heat loads.

Fig 4.10 to Fig 4.12 are shows the volume fraction contours of acetone-liquid at different heat loads ie $9 \mathrm{~W}, 12 \mathrm{~W}$ and $15 \mathrm{~W}$ respectively. It observed from these volume fraction contours is as time step size increases the volume fraction of acetone-liquid decreases when heat load given at evaporator section. And also observed that the acetone-liquid volume fraction decreases as heat load increases in the PHP.



Fig 4.7



Fig4.10

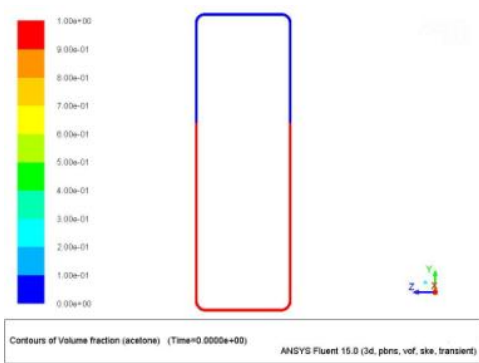

Fig4.8



Fig4.11

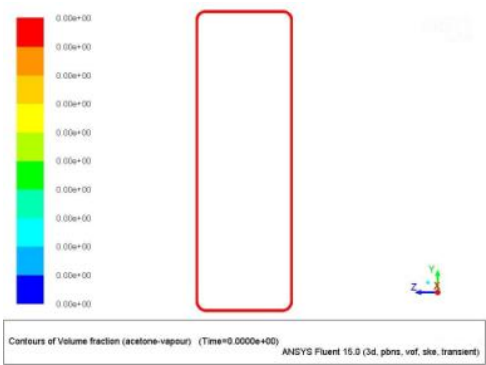

Fig4.9

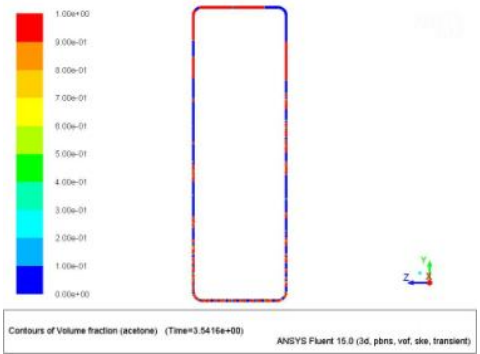

Fig4.12

\subsection{Temperature Contours of PHP:}

Fig 4.13 to Fig 4.18 shows the temperature contours of PHP at different volume fractions, different time step size by varying heat load. But the Fig 4.17 \& Fig 4.18 shows the Temperature contours of $80 \%$ volume fraction at $12 \mathrm{~W}$ heat load at different time steps. As time step increases acetone- liquid volume fraction becomes zero and acetone-vapour is only fluid which flows in the PHP. At this time temperature in the evaporator section is gradually increases and the evaporator section is maintained at same temperature. This Temperature contours shown in Fig 4.18.

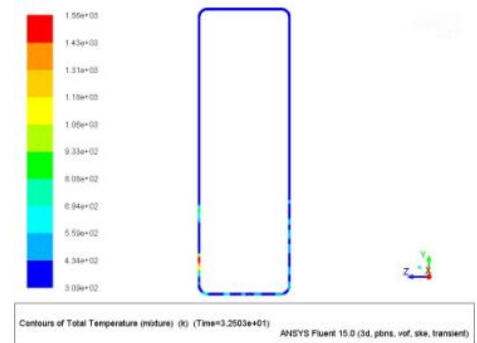

Fig4.13

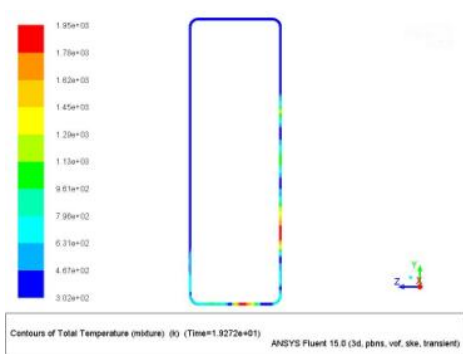

Fig4.16

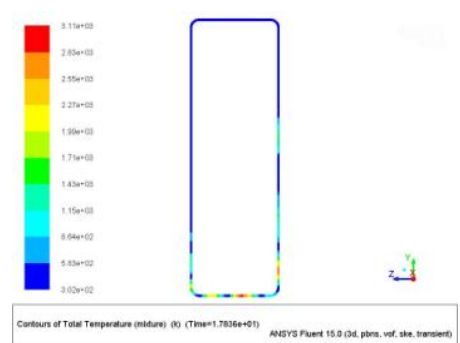

Fig4.14

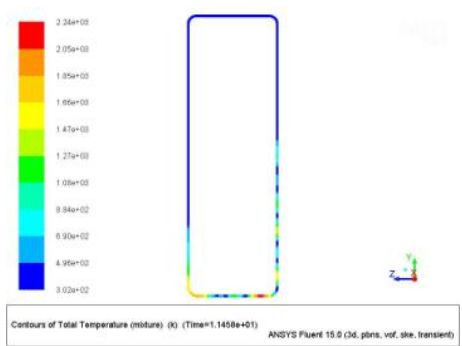

Fig4.15

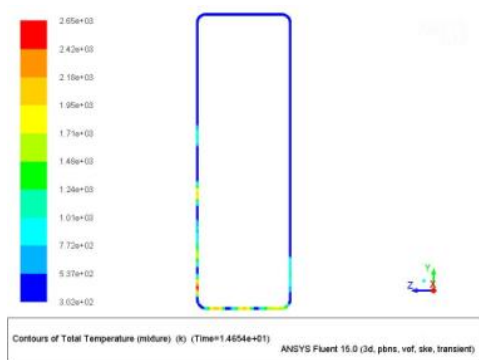

Fig4.17

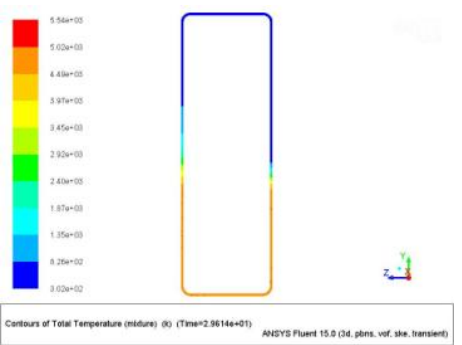

Fig4.18 


\section{Conclusion}

- In this present work based on CFD analysis of PHP it can be concluded that three phase flow is successfully simulated in FLUENT 15.0.

- From the results it is concluded that all working fluids are carrying heat from evaporator region to the condenser region by observing temperature change and flow of working fluids. Thus it can be concluded that the performance of PHP is primarily based on the phase change phenomenon.

- In CFD analysis the variation in evaporator and condenser wall temperatures with flow time is observed.

- It is observed that the thermal resistance decreases and heat transfer coefficient increases with increase in heat flux.

- CFD results are compared with available experimental base paper results. And acetone at $60 \%$ volume fraction is observed to be more suitable for PHP operation under different heat loads.

- At a fill ratio of $60 \%$, the PHP is exhibiting better heat transfer characteristics.

[1] Akachi,H(1990). Structure of Heat Pipe. US patent,4921041

[2] Cai,,C.LChen,J.F.Asfia(2006)Operating Characteristics Investigations in Pulsating Heat Pipe.Journal of Heat Transfer 128(12),1329-1334

[3] Charoensawan,P.S.Khandekar, M.Groll and P.Terdtoon(2001).Closed loop Pulsating Heat Pipes: Parametric Experimental Investigations. Applied Thermal Engineering 23(6),2009-2020

[4] P.S.Khandekar(2004)ThermoHydro Dynamics of Heat Pipes.PhD Dissertation, University of Stuggart, Germany.

[5] Meena,P.S .Rittidesch and P.Tammasaeng(2008).Effect of Inner Diameter and Inclination Angles on Operating Limits of Closed Loop Oscillating heat pipes with Check valves. American Journal of Applied Science1(2),100-103

[6] P.S.Khandekar(2008)Multiple Quasi Steady States in CLPHP.2 ${ }^{\text {nd }}$ Joint Workshop in Mechanical,Aerospace and Industrial Engineering ,IIT Kanpur, India

[7] Liu,S.J.Li, X.Dong and H.Chen(2007) Experimental Study of Closed Loop Pulsating Heat Pipes. Applied Thermal Engineering $128(1), 49-59$

[8] R.Naik, V.Varadrajan, G.Pundarika and K.R. Narasimha(2009) Experimental Investigation and Performane Evaluation of Closed Loop Pulsating Heat Pipe. Journal of Applied Fluid Mechanics 5(2-10) 